CRYSTALLOGRAPHIC COMMUNICATIONS

ISSN 2056-9890

Received 10 August 2016

Accepted 30 September 2016

Edited by M. Gdaniec, Adam Mickiewicz

University, Poland

Keywords: crystal structure; thiazine

compound; envelope pucker.

CCDC reference: 1507647

Supporting information: this article has supporting information at journals.iucr.org/e

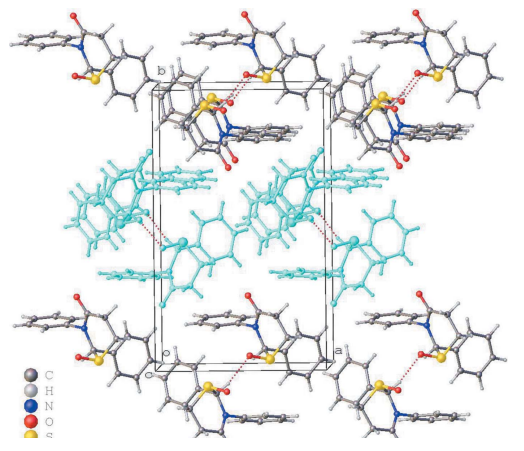

OPEN $\odot$ ACCESS

\section{Crystal structure of rac-2,3-diphenyl-2,3,5,6-tetra- hydro-4H-1,3-thiazin-4-one 1-oxide}

\author{
Hemant P. Yennawar, ${ }^{a}{\text { Ziwei } \text { Yang }^{\mathrm{b}} \text { and Lee J. Silverberg }}^{\mathrm{b} *}$ \\ a'Department of Biochemistry and Molecular Biology, Pennsylvania State University, University Park, PA 16802, USA, and \\ bennsylvania State University, Schuylkill Campus, 200 University Drive, Schuylkill Haven, PA 17972, USA. \\ *Correspondence e-mail: ljs43@psu.edu
}

In the title compound, $\mathrm{C}_{16} \mathrm{H}_{15} \mathrm{NO}_{2} \mathrm{~S}$ [alternative name: rac-2,3-diphenyl-1,3thiazinan-4-one 1-oxide], the thiazine ring exhibits an envelope conformation, with the $\mathrm{S}$ atom forming the flap. The sulfoxide $\mathrm{O}$ atom is in a pseudo-axial position on the thiazine ring and is trans to the phenyl group on C-2. The phenyl rings form a dihedral angle of $89.47(19)^{\circ}$. In this racemate crystal, homochiral molecules form slabs parallel to (010) of thickness $b / 2$ which then stack with alternating chirality in the $b$-axis direction. The stacking is aided by edge-to-face interactions between the phenyl rings of racemic molecules. Within each of the single-enantiomer slabs, the molecules are held by $\mathrm{C}-$ $\mathrm{H}$... O-type interactions, with an $\mathrm{H} \cdots \mathrm{O}$ distance of $2.30 \AA$, forming infinite chains along the $c$-axis direction, as well by the edge-to-face interactions between phenyl rings of neighboring molecules in the $a$-axis direction.

\section{Chemical context}

1,3-Thiazin-4-ones are a group of six-membered heterocycles with a wide range of biological activity (Ryabukhin et al., 1996). Surrey's research (Surrey et al., 1958; Surrey, 1963a) resulted in the discovery of two drugs, the anti-anxiety and muscle relaxant chlormezanone [2-(4-chlorophenyl)-3-methyl2,3,5,6-tetrahydro-4H-1,3-thiazin-4-one 1,1-dioxide; Merck Index, 2006; Tanaka \& Hirayama, 2005] and muscle relaxant dichlormezanone [2-(3,4-dichlorophenyl)-3-methyl-2,3,5,6tetrahydro-4H-1,3-thiazin-4-one 1,1-dioxide; Dictionary of Drugs, 1990]. These sulfones showed greater activity than the sulfides from which they were synthesized (Surrey et al., 1958). Surrey also prepared a variety of other sulfoxides and sulfones of 3-alkyl-2-aryl-2,3,5,6-tetrahydro-4H-1,3-thiazin-4-ones (Surrey, 1963a,b). Surrey did not successfully synthesize any 2-aryl-3-aryl-2,3,5,6-tetrahydro-4H-1,3-thiazin-4-ones (Silverberg et al., 2015), and to the best of our knowledge nobody has reported any oxides of this type of compound. We previously reported the crystal structure of 2,3-diphenyl-2,3,5,6-tetrahydro-4H-1,3-thiazin-4-one (Yennawar \& Silverberg, 2014). Herein, we report the crystal structure of that compound's sulfoxide, prepared using the method we have previously reported for oxidation of the five-membered 1,3-thiazolidin-4ones (Cannon et al., 2015).

\section{Structural commentary}

The crystal structure of this racemic compound shows a thiazine ring in an envelope pucker with puckering amplitude of 0.718 (3) $\AA$ (Fig. 1). The oxygen on sulfur is pseudo-axial on 
the thiazine ring. The two phenyl rings, on two adjacent atoms of the thiazine ring, are perpendicular to each other with an angle of $89.47(19)^{\circ}$ between their planes. The oxygen on sulfur and the phenyl ring on $\mathrm{C} 2$ are trans to each other.<smiles>O=C1CCS(=O)C(c2ccccc2)N1c1ccccc1</smiles>

\section{Supramolecular features}

The crystal consists of a racemic mixture of the title compound. The two phenyl groups and one of the two oxygen atoms participate in intermolecular interactions (Table 1). The molecules of single chirality form slabs in the $a c$ plane aided by $\pi-\pi$ edge-to-face interactions, with inter-centroid distance of 5.195 (3) $\AA$, in the $a$-axis direction and with $\mathrm{C}-\mathrm{H} \cdots \mathrm{O}$ hydrogen-bonds (Table 1) in the $c$-axis direction (Fig. 2). Along the $b$-axis direction, these slabs stack with alternating chirality, stabilized once again by $\pi-\pi$ edge-to-face interactions with inter-centroid distances of 5.021 (3) $\AA$.

\section{Database survey}

Crystal structures of a number of 1,3-thiazolidin-4-one 1-oxides have been reported (Wang et al., 2010; Johnson et al., 1983; Chen et al., 2011; Colombo et al., 2008; Yennawar et al., 2015); in each case the oxygen on sulfur and the group on C-2 had a trans relationship, as does the structure reported here. The structure of chlormezanone [2-(4-chlorophenyl)-3methyl-2,3,5,6-tetrahydro-4H-1,3-thiazin-4-one 1,1-dioxide] has also been disclosed (Tanaka \& Horayama, 2005). To the best of our knowledge, there have been no published

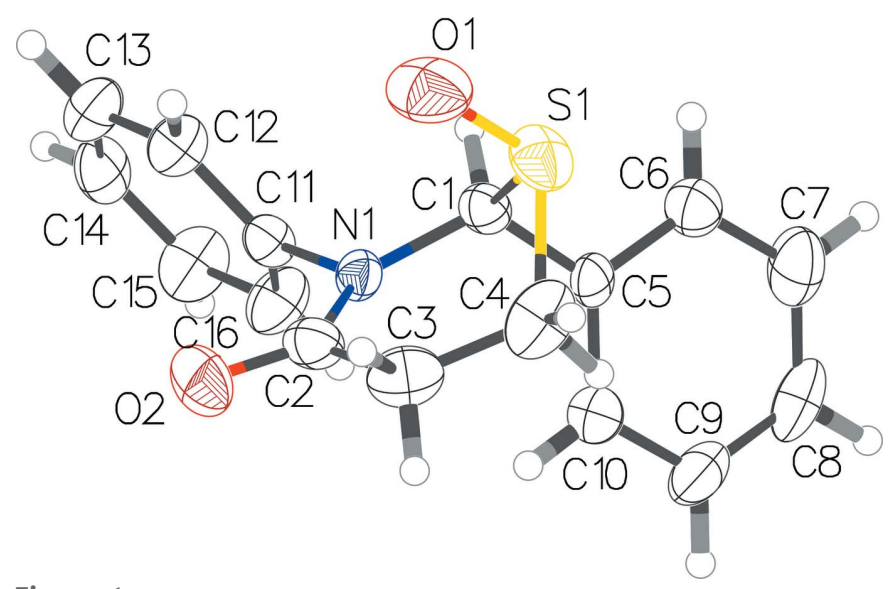

Figure 1

The molecular conformation and atom-numbering scheme for the title compound, with non- $\mathrm{H}$ atoms shown as $50 \%$ probability displacement ellipsoids.
Table 1

Hydrogen-bond geometry $\left(\AA{ }^{\circ}\right)$.

\begin{tabular}{lllll}
\hline$D-\mathrm{H} \cdots A$ & $D-\mathrm{H}$ & $\mathrm{H} \cdots A$ & $D \cdots A$ & $D-\mathrm{H} \cdots A$ \\
\hline $\mathrm{C} 1-\mathrm{H} 1 \cdots \mathrm{O} 1^{\mathrm{i}}$ & 0.98 & 2.30 & $3.261(5)$ & 167 \\
\hline
\end{tabular}

Symmetry code: (i) $-x,-y+1, z-\frac{1}{2}$.

crystal structures of a 2,3,5,6-tetrahydro-4H-1,3-thiazin-4-one 1-oxide.

\section{Synthesis and crystallization}

A $5 \mathrm{~mL}$ round-bottom flask was charged with $50.5 \mathrm{mg}$ of 2,3diphenyl-2,3,5,6-tetrahydro-4H-1,3-thiazin-4-one and $1.5 \mathrm{~mL}$ of methanol and stirred. A solution of $85.6 \mathrm{mg}$ Oxone ${ }^{\circledR}$ and $0.74 \mathrm{~mL}$ distilled water was added dropwise and the mixture was stirred until the reaction was complete as determined by TLC. The solids were dissolved by addition of $7.4 \mathrm{~mL}$ distilled water. The solution was extracted with $7.4 \mathrm{~mL}$ dichloromethane. The organic layer was washed with distilled water and then with sat. sodium chloride. The solution was dried over $\mathrm{Na}_{2} \mathrm{SO}_{4}$ and concentrated under vacuum to a crude solid. This was chromatographed on flash silica gel, eluting with $70 \%$ ethyl acetate/hexanes, $100 \%$ ethyl acetate, and $100 \%$ acetone, giving $37.5 \mathrm{mg}$ product (70\% yield), m.p.: $396-400 \mathrm{~K}, R_{f}=0.23$ (EtOAc). Crystals for X-ray crystallography were grown by slow evaporation from ethanol solution.

\section{Refinement}

Crystal data, data collection and structure refinement details are summarized in Table 2. The hydrogen atoms were placed

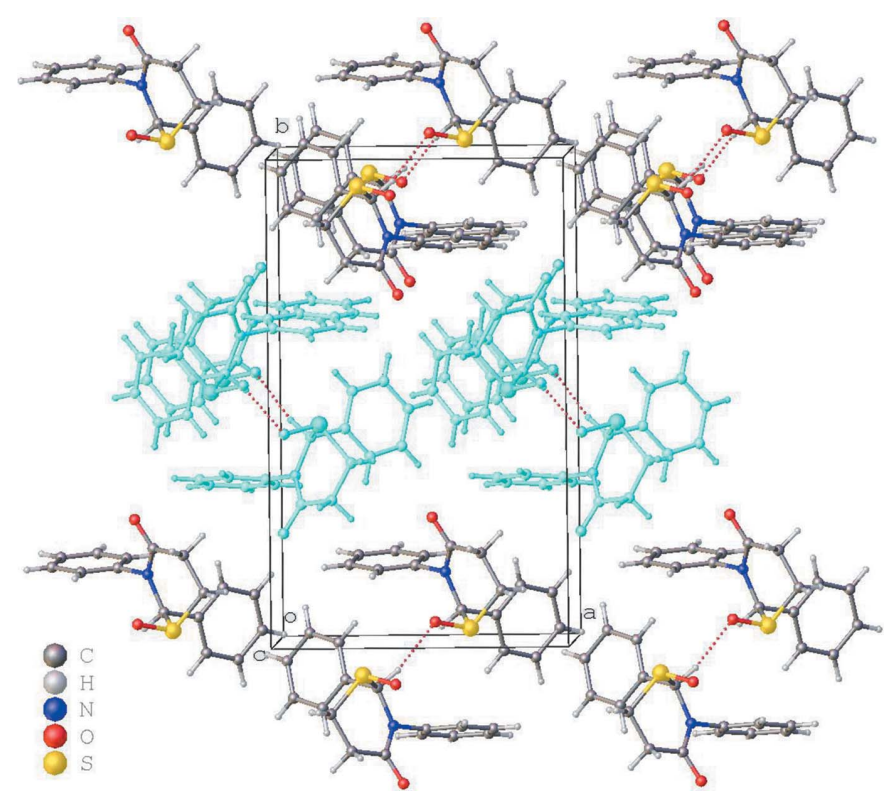

Figure 2

Packing viewed down the $c$ axis. Alternating slabs of enantiomers along the $b$-axis direction are differentiated by the color scheme. 
geometrically and allowed to ride on the carbon atoms during refinement, with $\mathrm{C}-\mathrm{H}$ distances of $0.98 \AA$ (methine), $0.96 \AA$ (methyl) and $0.93 \AA$ (aromatic) and with $U_{\text {iso }}(\mathrm{H})=$ $1.2 U_{\text {eq }}$ (aromatic and methine $\mathrm{C}$ ) or $1.5 U_{\text {eq }}$ (methyl).

\section{Acknowledgements}

We thank Penn State Schuylkill for financial support and NSF funding (CHEM-0131112) for the X-ray diffractometer.

\section{References}

Bruker (2001). SMART, SAINT and SADABS. Bruker AXS Inc., Madison, Wisconsin, USA.

Cannon, K., Gandla, D., Lauro, S., Silverberg, L., Tierney, J. \& Lagalante, A. (2015). Int. J. Chem. (Toronto, ON, Can.), 7(2), 7384.

Chen, H., Zai-Hong, G., Qing-Mei, Y. \& Xiao-Liu, L. (2011). Chin. J. Org. Chem. 31, 249-255.

Colombo, A., Fernàndez, J. C., Fernández-Forner, D., de la Figuera, N., Albericio, F. \& Forns, P. (2008). Tetrahedron Lett. 49, 1569-1572.

Dictionary of Drugs (1990). Edited by J. Elks \& C. R. Ganellin, p. 382. Cambridge: Chapman and Hall.

Dolomanov, O. V., Bourhis, L. J., Gildea, R. J., Howard, J. A. K. \& Puschmann, H. (2009). J. Appl. Cryst. 42, 339-341.

Flack, H. D. (1983). Acta Cryst. A39, 876-881.

Johnson, M. R., Fazio, M. J., Ward, D. L. \& Sousa, L. R. (1983). J. Org. Chem. 48, 494-499.

Merck Index (2006). 14th ed., edited by M. J. O'Neil, p. 349. Whitehouse Station: Merck \& Co.

Ryabukhin, Y. I., Korzhavina, O. B. \& Suzdalev, K. F. (1996). $A d v$. Heterocycl. Chem. 66, 131-190.

Sheldrick, G. M. (2008). Acta Cryst. A64, 112-122.

Sheldrick, G. M. (2015). Acta Cryst. C71, 3-8.

Silverberg, L. J., Pacheco, C. N., Lagalante, A., Cannon, K. C., Bachert, J. T., Xie, Y., Baker, L. \& Bayliff, J. A. (2015). Int. J. Chem. (Toronto, ON, Can.), 7(2), 150-162.

Surrey, A. R. (1963a). US Patent 3082209.

Surrey, A. R. (1963b). US Patent 3093639.

Surrey, A. R., Webb, W. G. \& Gesler, R. M. (1958). J. Am. Chem. Soc. 80, 3469-3471.
Table 2

Experimental details.

\begin{tabular}{|c|c|}
\hline \multicolumn{2}{|l|}{ Crystal data } \\
\hline Chemical formula & $\mathrm{C}_{16} \mathrm{H}_{15} \mathrm{NO}_{2} \mathrm{~S}$ \\
\hline$M_{\mathrm{r}}$ & 285.35 \\
\hline Crystal system, space group & Orthorhombic, Pna $_{1}$ \\
\hline Temperature (K) & 298 \\
\hline$a, b, c(\AA)$ & $10.547(4), 17.317(6), 7.592(3)$ \\
\hline$V\left(\AA^{3}\right)$ & $1386.5(8)$ \\
\hline$Z$ & 4 \\
\hline Radiation type & Мo $K \alpha$ \\
\hline$\mu\left(\mathrm{mm}^{-1}\right)$ & 0.23 \\
\hline Crystal size $(\mathrm{mm})$ & $0.23 \times 0.18 \times 0.16$ \\
\hline \multicolumn{2}{|l|}{ Data collection } \\
\hline Diffractometer & $\begin{array}{l}\text { Bruker SMART APEX CCD area } \\
\text { detector }\end{array}$ \\
\hline Absorption correction & $\begin{array}{l}\text { Multi-scan (SADABS; Bruker, } \\
\text { 2001) }\end{array}$ \\
\hline$T_{\min }, T_{\max }$ & $0.636,0.964$ \\
\hline $\begin{array}{l}\text { No. of measured, independent and } \\
\text { observed }[I>2 \sigma(I)] \text { reflections }\end{array}$ & $11124,3428,3182$ \\
\hline$R_{\text {int }}$ & 0.032 \\
\hline$(\sin \theta / \lambda)_{\max }\left(\AA^{-1}\right)$ & 0.668 \\
\hline \multicolumn{2}{|l|}{ Refinement } \\
\hline$R\left[F^{2}>2 \sigma\left(F^{2}\right)\right], w R\left(F^{2}\right), S$ & $0.075,0.161,1.26$ \\
\hline No. of reflections & 3428 \\
\hline No. of parameters & 181 \\
\hline No. of restraints & 1 \\
\hline $\mathrm{H}$-atom treatment & H-atom parameters constrained \\
\hline$\Delta \rho_{\max }, \Delta \rho_{\min }\left(\mathrm{e} \AA^{-3}\right)$ & $0.38,-0.27$ \\
\hline Absolute structure & Flack (1983), 4716 Friedel pairs \\
\hline Absolute structure parameter & $0.12(14)$ \\
\hline
\end{tabular}

Computer programs: SMART and SAINT (Bruker, 2001), SHELXS97 (Sheldrick, 2008), SHELXL2014 (Sheldrick, 2015) and OLEX2 (Dolomanov et al., 2009).

Tanaka, R. \& Horayama, N. (2005). X-Ray Struct. Anal. Online, 21, $\mathrm{x} 57-\mathrm{x} 58$.

Wang, Q., Xu, Z. \& Sun, Y. (2010). Acta Cryst. E66, o1422.

Yennawar, H. P. \& Silverberg, L. J. (2014). Acta Cryst. E70, o133. Yennawar, H. P., Tierney, J., Hullihen, P. D. \& Silverberg, L. J. (2015). Acta Cryst. E71, 264-267. 


\section{supporting information}

Acta Cryst. (2016). E72, 1541-1543 [https://doi.org/10.1107/S2056989016015395]

\section{Crystal structure of rac-2,3-diphenyl-2,3,5,6-tetrahydro-4H-1,3-thiazin-4-one 1 - oxide}

\section{Hemant P. Yennawar, Ziwei Yang and Lee J. Silverberg}

Computing details

Data collection: SMART (Bruker, 2001); cell refinement: SAINT (Bruker, 2001); data reduction: SAINT (Bruker, 2001); program(s) used to solve structure: SHELXS97 (Sheldrick, 2008); program(s) used to refine structure: SHELXL2014 (Sheldrick, 2015); molecular graphics: OLEX2 (Dolomanov et al., 2009); software used to prepare material for publication: OLEX2 (Dolomanov et al., 2009).

rac-2,3-Diphenyl-2,3,5,6-tetrahydro-4H-1,3-thiazin-4-one 1-oxide

\section{Crystal data}

$\mathrm{C}_{16} \mathrm{H}_{15} \mathrm{NO}_{2} \mathrm{~S}$

$M_{r}=285.35$

Orthorhombic, Pna2 $_{1}$

$a=10.547$ (4) $\AA$

$b=17.317(6) \AA$

$c=7.592(3) \AA$

$V=1386.5(8) \AA^{3}$

$Z=4$

$F(000)=600$

\section{Data collection}

Bruker SMART APEX CCD area detector diffractometer

Radiation source: fine-focus sealed tube

Parallel,graphite monochromator

$\varphi$ and $\omega$ scans

Absorption correction: multi-scan

(SADABS; Bruker, 2001)

$T_{\min }=0.636, T_{\max }=0.964$

\section{Refinement}

Refinement on $F^{2}$

Least-squares matrix: full

$R\left[F^{2}>2 \sigma\left(F^{2}\right)\right]=0.075$

$w R\left(F^{2}\right)=0.161$

$S=1.26$

3428 reflections

181 parameters

1 restraint

Primary atom site location: structure-invariant direct methods
$D_{\mathrm{x}}=1.367 \mathrm{Mg} \mathrm{m}^{-3}$

Mo $K \alpha$ radiation, $\lambda=0.71073 \AA$

Cell parameters from 3661 reflections

$\theta=2.3-26.4^{\circ}$

$\mu=0.23 \mathrm{~mm}^{-1}$

$T=298 \mathrm{~K}$

Block, colorless

$0.23 \times 0.18 \times 0.16 \mathrm{~mm}$

11124 measured reflections

3428 independent reflections

3182 reflections with $I>2 \sigma(I)$

$R_{\text {int }}=0.032$

$\theta_{\max }=28.3^{\circ}, \theta_{\min }=2.3^{\circ}$

$h=-13 \rightarrow 13$

$k=-20 \rightarrow 23$

$l=-10 \rightarrow 10$

Secondary atom site location: difference Fourier map

Hydrogen site location: inferred from neighbouring sites

$\mathrm{H}$-atom parameters constrained

$w=1 /\left[\sigma^{2}\left(F_{\mathrm{o}}^{2}\right)+(0.0582 P)^{2}+0.8049 P\right]$

where $P=\left(F_{\mathrm{o}}^{2}+2 F_{\mathrm{c}}^{2}\right) / 3$

$(\Delta / \sigma)_{\max }<0.001$

$\Delta \rho_{\max }=0.38$ e $\AA^{-3}$

$\Delta \rho_{\min }=-0.27$ e $\AA^{-3}$ 
Absolute structure: Flack (1983), 4716 Friedel pairs

\section{Special details}

Experimental. 1. SADABS was used for absorption correction. R(int) was 0.0424 before and 0.0268 after correction. The Ratio of minimum to maximum transmission is 0.6364 . The $\lambda / 2$ correction factor is 0.0015 .

2. The data collection nominally covered a full sphere of reciprocal space by a combination of 4 sets of $\omega$ scans each set at different $\varphi$ and/or $2 \theta$ angles and each scan ( 5 s exposure) covering $-0.300^{\circ}$ degrees in $\omega$. The crystal to detector distance was $5.82 \mathrm{~cm}$.

Geometry. All esds (except the esd in the dihedral angle between two 1.s. planes) are estimated using the full covariance matrix. The cell esds are taken into account individually in the estimation of esds in distances, angles and torsion angles; correlations between esds in cell parameters are only used when they are defined by crystal symmetry. An approximate (isotropic) treatment of cell esds is used for estimating esds involving 1.s. planes.

Refinement. Refinement of $\mathrm{F}^{2}$ against ALL reflections. The weighted R-factor $\mathrm{wR}$ and goodness of fit $\mathrm{S}$ are based on $\mathrm{F}^{2}$, conventional R-factors $R$ are based on $F$, with $F$ set to zero for negative $F^{2}$. The threshold expression of $F^{2}>2$ sigma $\left(F^{2}\right)$ is used only for calculating R-factors(gt) etc. and is not relevant to the choice of reflections for refinement. R-factors based on $\mathrm{F}^{2}$ are statistically about twice as large as those based on $\mathrm{F}$, and $\mathrm{R}$ - factors based on ALL data will be even larger.

Fractional atomic coordinates and isotropic or equivalent isotropic displacement parameters $\left(\AA^{2}\right)$

\begin{tabular}{|c|c|c|c|c|}
\hline & $x$ & $y$ & $z$ & $U_{\text {iso }} * / U_{\text {eq }}$ \\
\hline $\mathrm{S} 1$ & $0.17133(10)$ & $0.45935(5)$ & $-1.97816(14)$ & $0.0469(3)$ \\
\hline $\mathrm{O} 1$ & 0.0575 & $0.44303(18)$ & $-1.8700(4)$ & $0.0659(9)$ \\
\hline $\mathrm{O} 2$ & $0.0431(3)$ & $0.23452(16)$ & $-2.0769(4)$ & $0.0542(8)$ \\
\hline N1 & $0.0796(2)$ & $0.34956(15)$ & $-2.2011(4)$ & $0.0281(6)$ \\
\hline $\mathrm{C} 1$ & $0.1326(3)$ & 0.42779 (19) & $-2.2029(4)$ & $0.0304(7)$ \\
\hline H1 & 0.0646 & 0.4619 & -2.2442 & $0.036^{*}$ \\
\hline $\mathrm{C} 2$ & $0.1038(4)$ & $0.2938(2)$ & $-2.0808(5)$ & $0.0375(8)$ \\
\hline $\mathrm{C} 3$ & $0.2109(4)$ & $0.3050(2)$ & $-1.9494(5)$ & $0.0483(10)$ \\
\hline $\mathrm{H} 3 \mathrm{~A}$ & 0.1771 & 0.2936 & -1.8334 & $0.058 *$ \\
\hline $\mathrm{H} 3 \mathrm{~B}$ & 0.2745 & 0.2660 & -1.9744 & $0.058^{*}$ \\
\hline $\mathrm{C} 4$ & $0.2783(4)$ & $0.3813(3)$ & $-1.9375(5)$ & $0.0521(11)$ \\
\hline $\mathrm{H} 4 \mathrm{~A}$ & 0.3465 & 0.3828 & -2.0231 & $0.063 *$ \\
\hline $\mathrm{H} 4 \mathrm{~B}$ & 0.3151 & 0.3870 & -1.8211 & $0.063 *$ \\
\hline $\mathrm{C} 5$ & $0.2437(3)$ & $0.44151(17)$ & $-2.3232(4)$ & $0.0278(6)$ \\
\hline C6 & $0.2671(4)$ & $0.5164(2)$ & $-2.3791(5)$ & $0.0392(8)$ \\
\hline H6 & 0.2126 & 0.5559 & -2.3446 & $0.047^{*}$ \\
\hline $\mathrm{C} 7$ & $0.3688(4)$ & $0.5333(3)$ & $-2.4840(8)$ & $0.0534(10)$ \\
\hline $\mathrm{H7}$ & 0.3842 & 0.5839 & -2.5186 & $0.064 *$ \\
\hline $\mathrm{C} 8$ & $0.4485(4)$ & $0.4748(3)$ & $-2.5384(6)$ & $0.0517(11)$ \\
\hline H8 & 0.5175 & 0.4860 & -2.6103 & $0.062 *$ \\
\hline C9 & 0.4261 & $0.4006(2)$ & $-2.4869(6)$ & $0.0472(9)$ \\
\hline H9 & 0.4799 & 0.3613 & -2.5247 & $0.057^{*}$ \\
\hline $\mathrm{C} 10$ & $0.3245(3)$ & $0.3830(2)$ & $-2.3791(5)$ & $0.0391(8)$ \\
\hline $\mathrm{H} 10$ & 0.3102 & 0.3323 & -2.3441 & $0.047^{*}$ \\
\hline C11 & $-0.0254(3)$ & $0.33887(18)$ & $-2.3192(4)$ & $0.0297(7)$ \\
\hline $\mathrm{C} 12$ & -0.1478 (4) & $0.3459(2)$ & $-2.2599(5)$ & $0.0408(8)$ \\
\hline H12 & -0.1627 & 0.3562 & -2.1415 & $0.049 *$ \\
\hline C13 & -0.2485 & $0.3378(2)$ & $-2.3733(6)$ & $0.0477(10)$ \\
\hline
\end{tabular}


supporting information

\begin{tabular}{lllll}
$\mathrm{H} 13$ & -0.3313 & 0.3424 & -2.3323 & $0.057^{*}$ \\
$\mathrm{C} 14$ & $-0.2254(4)$ & $0.3231(2)$ & $-2.5468(5)$ & $0.0439(9)$ \\
$\mathrm{H} 14$ & -0.2934 & 0.3171 & -2.6236 & $0.053^{*}$ \\
$\mathrm{C} 15$ & $-0.1043(4)$ & $0.3169(3)$ & $-2.6103(5)$ & $0.0492(10)$ \\
$\mathrm{H} 15$ & -0.0901 & 0.3077 & -2.7294 & $0.059^{*}$ \\
C16 & $-0.0032(4)$ & $0.3244(2)$ & $-2.4953(5)$ & $0.0454(9)$ \\
$\mathrm{H} 16$ & 0.0794 & 0.3197 & -2.5366 & $0.055^{*}$ \\
\hline
\end{tabular}

Atomic displacement parameters $\left(\AA^{2}\right)$

\begin{tabular}{lllllll}
\hline & $U^{11}$ & $U^{22}$ & $U^{33}$ & $U^{12}$ & $U^{13}$ & $U^{23}$ \\
\hline S1 & $0.0600(6)$ & $0.0427(5)$ & $0.0380(4)$ & $-0.0080(4)$ & $0.0072(5)$ & $-0.0128(4)$ \\
O1 & $0.083(2)$ & $0.0605(18)$ & $0.0548(19)$ & $0.0131(17)$ & $0.0259(18)$ & $-0.0026(16)$ \\
O2 & $0.0614(18)$ & $0.0366(15)$ & $0.0648(19)$ & $-0.0055(13)$ & $0.0023(15)$ & $0.0188(13)$ \\
N1 & $0.0242(13)$ & $0.0277(13)$ & $0.0325(13)$ & $-0.0015(10)$ & $0.0013(12)$ & $0.0054(11)$ \\
C1 & $0.0326(16)$ & $0.0262(15)$ & $0.0323(16)$ & $0.0012(13)$ & $-0.0013(14)$ & $0.0026(13)$ \\
C2 & $0.041(2)$ & $0.0306(17)$ & $0.0410(19)$ & $0.0064(14)$ & $0.0042(16)$ & $0.0056(15)$ \\
C3 & $0.049(2)$ & $0.055(2)$ & $0.041(2)$ & $0.0142(18)$ & $-0.0079(18)$ & $0.0038(18)$ \\
C4 & $0.042(2)$ & $0.080(3)$ & $0.034(2)$ & $-0.003(2)$ & $-0.0060(16)$ & $0.0044(18)$ \\
C5 & $0.0233(14)$ & $0.0287(14)$ & $0.0316(16)$ & $-0.0045(12)$ & $-0.0070(12)$ & $0.0007(13)$ \\
C6 & $0.0376(19)$ & $0.0357(17)$ & $0.0441(19)$ & $-0.0002(15)$ & $-0.0024(16)$ & $0.0036(16)$ \\
C7 & $0.046(2)$ & $0.057(2)$ & $0.057(2)$ & $-0.0126(18)$ & $0.003(3)$ & $0.018(2)$ \\
C8 & $0.0308(19)$ & $0.081(3)$ & $0.043(2)$ & $-0.017(2)$ & $0.0064(16)$ & $0.006(2)$ \\
C9 & $0.0275(16)$ & $0.064(2)$ & $0.051(2)$ & $-0.0013(16)$ & $0.0053(19)$ & $-0.012(2)$ \\
C10 & $0.0344(19)$ & $0.0370(17)$ & $0.046(2)$ & $-0.0008(14)$ & $-0.0017(16)$ & $-0.0019(17)$ \\
C11 & $0.0333(17)$ & $0.0266(14)$ & $0.0293(15)$ & $0.0000(12)$ & $-0.0056(14)$ & $-0.0002(13)$ \\
C12 & $0.0364(19)$ & $0.047(2)$ & $0.0388(19)$ & $-0.0003(16)$ & $0.0021(15)$ & $0.0013(17)$ \\
C13 & $0.0287(18)$ & $0.055(2)$ & $0.059(2)$ & $-0.0006(17)$ & $0.0004(18)$ & $-0.002(2)$ \\
C14 & $0.042(2)$ & $0.040(2)$ & $0.050(2)$ & $-0.0065(16)$ & $-0.0185(17)$ & $0.0007(17)$ \\
C15 & $0.048(2)$ & $0.073(3)$ & $0.0261(17)$ & $-0.004(2)$ & $-0.0027(17)$ & $-0.0071(18)$ \\
C16 & $0.0369(18)$ & $0.062(2)$ & $0.0374(19)$ & $-0.0022(16)$ & $0.0089(17)$ & $-0.0001(19)$ \\
& & & & & &
\end{tabular}

Geometric parameters ( $\left.\AA,{ }^{\circ}\right)$

\begin{tabular}{llll}
\hline $\mathrm{S} 1-\mathrm{O} 1$ & $1.482(3)$ & $\mathrm{C} 5-\mathrm{C} 10$ & $1.390(5)$ \\
$\mathrm{S} 1-\mathrm{C} 1$ & $1.838(4)$ & $\mathrm{C} 6-\mathrm{C} 7$ & $1.368(6)$ \\
$\mathrm{S} 1-\mathrm{C} 4$ & $1.787(5)$ & $\mathrm{C} 7-\mathrm{C} 8$ & $1.379(6)$ \\
$\mathrm{O} 2-\mathrm{C} 2$ & $1.210(5)$ & $\mathrm{C} 8-\mathrm{C} 9$ & $1.364(6)$ \\
$\mathrm{N} 1-\mathrm{C} 1$ & $\mathrm{C} 9-\mathrm{C} 10$ & $1.383(5)$ \\
$\mathrm{N} 1-\mathrm{C} 2$ & $1.466(4)$ & $\mathrm{C} 11-\mathrm{C} 12$ & $1.372(5)$ \\
$\mathrm{N} 1-\mathrm{C} 11$ & $1.354(4)$ & $\mathrm{C} 11-\mathrm{C} 16$ & $1.380(5)$ \\
$\mathrm{C} 1-\mathrm{C} 5$ & $1.436(4)$ & $\mathrm{C} 12-\mathrm{C} 13$ & $1.375(6)$ \\
$\mathrm{C} 2-\mathrm{C} 3$ & $1.505(5)$ & $\mathrm{C} 13-\mathrm{C} 14$ & $1.363(6)$ \\
$\mathrm{C} 3-\mathrm{C} 4$ & $1.519(5)$ & $\mathrm{C} 14-\mathrm{C} 15$ & $1.370(6)$ \\
$\mathrm{C} 5-\mathrm{C} 6$ & $1.503(6)$ & $\mathrm{C} 15-\mathrm{C} 16$ & $1.384(6)$ \\
& $1.387(5)$ & $\mathrm{C} 6-\mathrm{C} 5-\mathrm{C} 10$ & $118.7(3)$ \\
$\mathrm{O} 1-\mathrm{S} 1-\mathrm{C} 1$ & $106.12(18)$ & $\mathrm{C} 10-\mathrm{C} 5-\mathrm{C} 1$ & $123.2(3)$ \\
$\mathrm{O} 1-\mathrm{S} 1-\mathrm{C} 4$ & $105.8(2)$ & &
\end{tabular}




\begin{tabular}{|c|c|c|c|}
\hline $\mathrm{C} 4-\mathrm{S} 1-\mathrm{C} 1$ & $94.34(17)$ & $\mathrm{C} 7-\mathrm{C} 6-\mathrm{C} 5$ & $121.1(4)$ \\
\hline $\mathrm{C} 2-\mathrm{N} 1-\mathrm{C} 1$ & $126.4(3)$ & $\mathrm{C} 6-\mathrm{C} 7-\mathrm{C} 8$ & $119.7(4)$ \\
\hline $\mathrm{C} 2-\mathrm{N} 1-\mathrm{C} 11$ & $118.4(3)$ & $\mathrm{C} 9-\mathrm{C} 8-\mathrm{C} 7$ & $120.1(4)$ \\
\hline $\mathrm{C} 11-\mathrm{N} 1-\mathrm{C} 1$ & $114.0(2)$ & $\mathrm{C} 8-\mathrm{C} 9-\mathrm{C} 10$ & $120.7(4)$ \\
\hline $\mathrm{N} 1-\mathrm{C} 1-\mathrm{S} 1$ & $110.6(2)$ & $\mathrm{C} 9-\mathrm{C} 10-\mathrm{C} 5$ & $119.7(3)$ \\
\hline $\mathrm{N} 1-\mathrm{C} 1-\mathrm{C} 5$ & $116.7(3)$ & $\mathrm{C} 12-\mathrm{C} 11-\mathrm{N} 1$ & $120.6(3)$ \\
\hline $\mathrm{C} 5-\mathrm{C} 1-\mathrm{S} 1$ & $110.1(2)$ & $\mathrm{C} 12-\mathrm{C} 11-\mathrm{C} 16$ & $119.5(3)$ \\
\hline $\mathrm{O} 2-\mathrm{C} 2-\mathrm{N} 1$ & $121.4(3)$ & $\mathrm{C} 16-\mathrm{C} 11-\mathrm{N} 1$ & $119.8(3)$ \\
\hline $\mathrm{O} 2-\mathrm{C} 2-\mathrm{C} 3$ & $119.0(3)$ & $\mathrm{C} 11-\mathrm{C} 12-\mathrm{C} 13$ & $120.8(4)$ \\
\hline $\mathrm{N} 1-\mathrm{C} 2-\mathrm{C} 3$ & $119.5(3)$ & $\mathrm{C} 14-\mathrm{C} 13-\mathrm{C} 12$ & $119.1(4)$ \\
\hline $\mathrm{C} 4-\mathrm{C} 3-\mathrm{C} 2$ & $120.2(3)$ & $\mathrm{C} 13-\mathrm{C} 14-\mathrm{C} 15$ & $121.4(4)$ \\
\hline $\mathrm{C} 3-\mathrm{C} 4-\mathrm{S} 1$ & $110.9(3)$ & $\mathrm{C} 14-\mathrm{C} 15-\mathrm{C} 16$ & $119.3(4)$ \\
\hline $\mathrm{C} 6-\mathrm{C} 5-\mathrm{C} 1$ & $118.1(3)$ & $\mathrm{C} 11-\mathrm{C} 16-\mathrm{C} 15$ & $119.9(3)$ \\
\hline $\mathrm{S} 1-\mathrm{C} 1-\mathrm{C} 5-\mathrm{C} 6$ & $-76.3(3)$ & $\mathrm{C} 2-\mathrm{N} 1-\mathrm{C} 11-\mathrm{C} 16$ & $-109.6(4)$ \\
\hline $\mathrm{S} 1-\mathrm{C} 1-\mathrm{C} 5-\mathrm{C} 10$ & $102.9(3)$ & $\mathrm{C} 2-\mathrm{C} 3-\mathrm{C} 4-\mathrm{S} 1$ & $34.5(4)$ \\
\hline $\mathrm{O} 1-\mathrm{S} 1-\mathrm{C} 1-\mathrm{N} 1$ & $-49.2(3)$ & $\mathrm{C} 4-\mathrm{S} 1-\mathrm{C} 1-\mathrm{N} 1$ & $58.6(3)$ \\
\hline $\mathrm{O} 1-\mathrm{S} 1-\mathrm{C} 1-\mathrm{C} 5$ & $-179.5(2)$ & $\mathrm{C} 4-\mathrm{S} 1-\mathrm{C} 1-\mathrm{C} 5$ & $-71.7(3)$ \\
\hline $\mathrm{O} 1-\mathrm{S} 1-\mathrm{C} 4-\mathrm{C} 3$ & $46.9(3)$ & $\mathrm{C} 5-\mathrm{C} 6-\mathrm{C} 7-\mathrm{C} 8$ & $1.3(7)$ \\
\hline $\mathrm{O} 2-\mathrm{C} 2-\mathrm{C} 3-\mathrm{C} 4$ & $-173.1(4)$ & $\mathrm{C} 6-\mathrm{C} 5-\mathrm{C} 10-\mathrm{C} 9$ & $0.6(5)$ \\
\hline $\mathrm{N} 1-\mathrm{C} 1-\mathrm{C} 5-\mathrm{C} 6$ & $156.6(3)$ & $\mathrm{C} 6-\mathrm{C} 7-\mathrm{C} 8-\mathrm{C} 9$ & $-0.3(7)$ \\
\hline $\mathrm{N} 1-\mathrm{C} 1-\mathrm{C} 5-\mathrm{C} 10$ & $-24.1(4)$ & $\mathrm{C} 7-\mathrm{C} 8-\mathrm{C} 9-\mathrm{C} 10$ & $-0.5(7)$ \\
\hline $\mathrm{N} 1-\mathrm{C} 2-\mathrm{C} 3-\mathrm{C} 4$ & $8.0(5)$ & $\mathrm{C} 8-\mathrm{C} 9-\mathrm{C} 10-\mathrm{C} 5$ & $0.3(6)$ \\
\hline $\mathrm{N} 1-\mathrm{C} 11-\mathrm{C} 12-\mathrm{C} 13$ & $178.2(3)$ & $\mathrm{C} 10-\mathrm{C} 5-\mathrm{C} 6-\mathrm{C} 7$ & $-1.4(6)$ \\
\hline $\mathrm{N} 1-\mathrm{C} 11-\mathrm{C} 16-\mathrm{C} 15$ & $-177.7(3)$ & $\mathrm{C} 11-\mathrm{N} 1-\mathrm{C} 1-\mathrm{S} 1$ & $139.1(2)$ \\
\hline $\mathrm{C} 1-\mathrm{S} 1-\mathrm{C} 4-\mathrm{C} 3$ & $-61.2(3)$ & $\mathrm{C} 11-\mathrm{N} 1-\mathrm{C} 1-\mathrm{C} 5$ & $-94.1(3)$ \\
\hline $\mathrm{C} 1-\mathrm{N} 1-\mathrm{C} 2-\mathrm{O} 2$ & $170.2(3)$ & $\mathrm{C} 11-\mathrm{N} 1-\mathrm{C} 2-\mathrm{O} 2$ & $3.5(5)$ \\
\hline $\mathrm{C} 1-\mathrm{N} 1-\mathrm{C} 2-\mathrm{C} 3$ & $-11.0(5)$ & $\mathrm{C} 11-\mathrm{N} 1-\mathrm{C} 2-\mathrm{C} 3$ & $-177.6(3)$ \\
\hline $\mathrm{C} 1-\mathrm{N} 1-\mathrm{C} 11-\mathrm{C} 12$ & $-95.3(4)$ & $\mathrm{C} 11-\mathrm{C} 12-\mathrm{C} 13-\mathrm{C} 14$ & $-0.3(6)$ \\
\hline $\mathrm{C} 1-\mathrm{N} 1-\mathrm{C} 11-\mathrm{C} 16$ & $82.2(4)$ & $\mathrm{C} 12-\mathrm{C} 11-\mathrm{C} 16-\mathrm{C} 15$ & $-0.1(6)$ \\
\hline $\mathrm{C} 1-\mathrm{C} 5-\mathrm{C} 6-\mathrm{C} 7$ & $177.9(4)$ & $\mathrm{C} 12-\mathrm{C} 13-\mathrm{C} 14-\mathrm{C} 15$ & $-0.6(6)$ \\
\hline $\mathrm{C} 1-\mathrm{C} 5-\mathrm{C} 10-\mathrm{C} 9$ & $-178.7(3)$ & $\mathrm{C} 13-\mathrm{C} 14-\mathrm{C} 15-\mathrm{C} 16$ & $1.1(7)$ \\
\hline $\mathrm{C} 2-\mathrm{N} 1-\mathrm{C} 1-\mathrm{S} 1$ & $-28.1(4)$ & $\mathrm{C} 14-\mathrm{C} 15-\mathrm{C} 16-\mathrm{C} 11$ & $-0.8(6)$ \\
\hline $\mathrm{C} 2-\mathrm{N} 1-\mathrm{C} 1-\mathrm{C} 5$ & $98.7(4)$ & $\mathrm{C} 16-\mathrm{C} 11-\mathrm{C} 12-\mathrm{C} 13$ & $0.7(6)$ \\
\hline $\mathrm{C} 2-\mathrm{N} 1-\mathrm{C} 11-\mathrm{C} 12$ & $72.9(4)$ & & \\
\hline
\end{tabular}

Hydrogen-bond geometry $\left(\AA,{ }^{\circ}\right)$

\begin{tabular}{lllll}
\hline$D-\mathrm{H} \cdots A$ & $D-\mathrm{H}$ & $\mathrm{H} \cdots A$ & $D \cdots A$ & $D-\mathrm{H} \cdots A$ \\
\hline $\mathrm{C} 1-\mathrm{H} 1 \cdots \mathrm{O} 1^{\mathrm{i}}$ & 0.98 & 2.30 & $3.261(5)$ & 167 \\
\hline
\end{tabular}

Symmetry code: (i) $-x,-y+1, z-1 / 2$. 